\title{
Multi-channel switch state and wireless detection system design Limin $\mathrm{Liu}^{1, \mathrm{a}}$, Hongbin $\mathrm{Yu}^{2, \mathrm{~b}}$ \\ ${ }^{1}$ School of Mechanical Engineering ,Tianjin Polytechnic University,Tianjin,300387, China; \\ ${ }^{2}$ School of Mechanical Engineering ,Tianjin Polytechnic University,Tianjin,300387, China. \\ a981363668@qq.com, bhbyu@yahoo.cn
}

Keywords: switch status; wireless communications; real-time detection; low power consumption.

\begin{abstract}
Multi-channel switch state detecting apparatus for wireless communication can simultaneously detect multiple switch status, real-time detection of multiple switch status. When the switch does not move, switch status detection means dormant. When the switch has the operation, switch status acquisition device evoked potential changes, wake the entire device, thereby reducing the power consumption of the entire system, to achieve green, low power consumption.
\end{abstract}

\section{The introduction}

In industrial production, we need to understand the equipment working station, in order to better control the production process. In order to be able to real-time and convenient testing equipment status, there are many "on" or "off" position signal, known as the "on-off" professional[1] , in the actual production need for some switch state for testing signal. Traditional detection methods need to access within the system under test instrument and signal lines need to tear open which may result in switch short circuit or open circuit, reducing the safety of the whole production system stability. Secondly.in the actual production site wiring construction, construction cost is higher, the construction cycle is long, with the problems of common operation instability and poor reliability. To solve the problems above, in the various steps of the switch quantity processing of real-time and on the basis of detailed analysis, this paper proposes a multi-channel switch state detection based on wireless transmission system, the implementation of the scheme.

\section{Multi-channel switch state detection system analysis}

Multi-channel switch state detection system is for the real-time collection, transmission, testing and storage of multi-channel switch state. Figure 1 simplified diagram for the system working process.

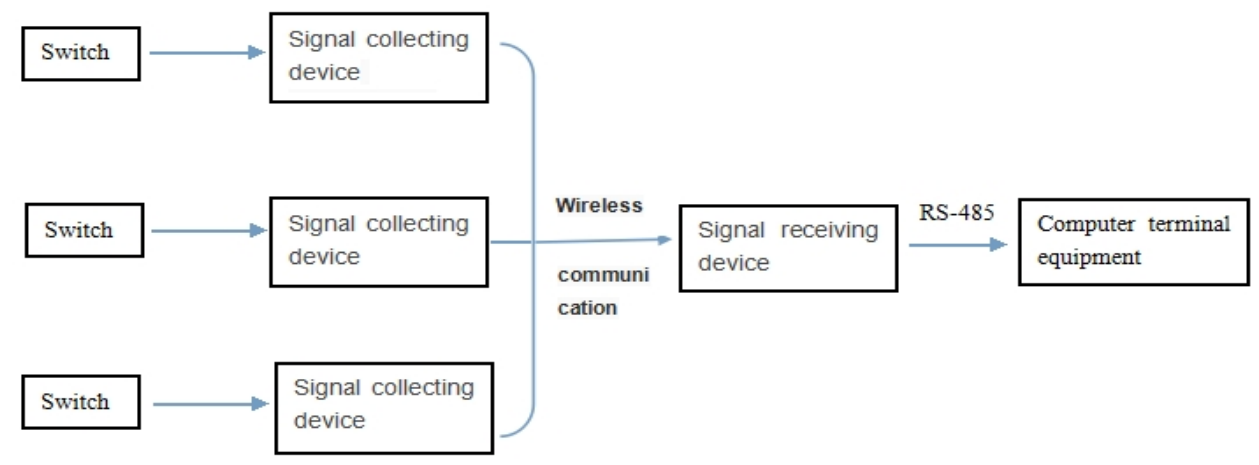

Figure 1.System workflow simplified diagram

Based on the market for mechanical contact switch when switch on the mechanical contact switch in action occurs, due to the effect of elasticity of the mechanical contact it will not immediately connect when a contact switch is closed steady on, also won't instantaneous disconnect when disconnected. Thus in the instant of closing and opening are accompanied by a series of jitter, are shown in figure 2 below. The length of shaking time is determined by the mechanical 
characteristics of the contact, generally for $5 \sim 10 \mathrm{~ms}$ [2]. In order to guarantee the accuracy of the system acquisition switch status signal, we need to eliminate the shake of the switch contact. Commonly we use shaking processing method with software and hardware such two elimination method. Hardware shaking commonly use double shake elimination and filtering method.Filtering away shaking method use the methods of shunt capacitance, with the use of capacitor discharge delay, to realize the hardware shake. Shake circuit is shown in figure 3. If the switch is more, we commonly use software method to shake, which detects the contact closure after executing a delay program, $5 \sim 10 \mathrm{~ms}$ delay, letting the frontier dithering disappeared again after inspecting the status of contact, and if you still keep close state level, it will be recognized as a real contact press. When detected by contact after their release, also for $5 \sim 10$ ms delay, after being disappeared along the jitter after processing program can be transferred to the contact. This system uses the software shaking method on the basis of the guarantee of the accuracy and simple of the circuit.

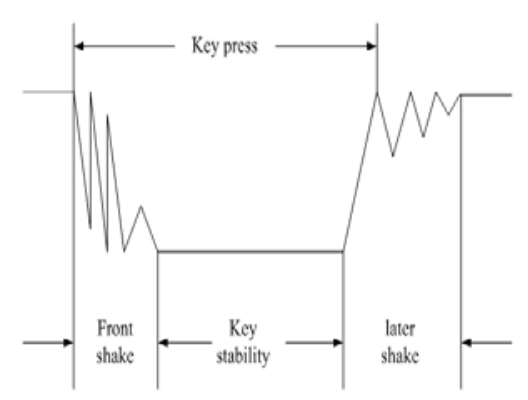

Figure 2. Switch shake

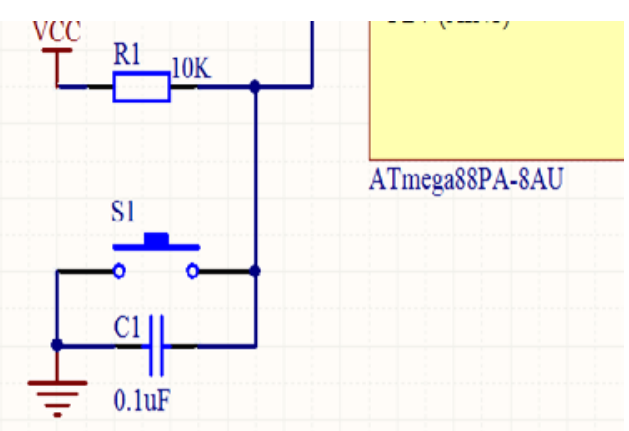

Figure 3. Hardware debounced

Wireless communication in common use such as $2.4 \mathrm{GHz}$ communication and $433 \mathrm{MHZ}$ communication technology[3]. This system using wireless transmission way is $433 \mathrm{MHZ}$ communication protocol. $433 \mathrm{MHZ}$ wireless communication, penetrating power stronger than 2.4Ghz wireless communication, environment adaptable; Strong anti-interference ability than the 2.4Ghz wireless communication. Currently in restaurant wireless order, car remote control lock, intelligent building wireless temperature measurement are the application of $433 \mathrm{MHZ}$ wireless communication products. This system through the analysis and the scene debugging, to ensure the stability of data transmission, using the $433 \mathrm{MHZ}$ communication.

\section{Design}

\subsection{Multiplexer switch status detection collection device based on wireless communications}

Switch status acquisition device is detected by the micro switch installed by the magnet is close to determine switch status information. Switch status acquisition reed on the unit is a special kind of magnetic switch, its two contacts are made of special materials, are encapsulated in a vacuum glass tube. When the switch on miniature magnet near its end, the contacts will suck together, the circuit will turn on. When micro letter magnet end switch away from its contact opens, the circuit will be disconnected. SCM off by the detection circuit determine the switch status information. Collection device which uses a microcontroller ATMEGA88PA-AU model is a high performance, low-power 8-bit AVR microcontroller. Signal microcontroller will be collected, transmitted to the switch status detection receiver by wireless transmission module.

Switch status means low-power algorithm, the switch does not move, switch status detection means dormant. When the switch has the operation, switch status acquisition device evoked potential changes, waking the entire apparatus and the apparatus has a self-examination wake-up function, the default every 8s software means self-wake of switch status information for verification. In order not only to achieve low power consumption, but also to ensure the quasi-signal acquisition.

\subsection{Receiver based on wireless communications multiplexer switch status detection}

Switch status receiving device can receive simultaneous multi-channel digital status signals digital acquisition device transmitted and the same model is ATMEGA88PA-AU microprocessor. 
Switch status receiving device on a wireless communication module receives the switch status switch status information acquisition device to achieve real-time transmission and reception switch status via wireless transmission format. Switch status information receiving apparatus switch status via RS-485 serial cable to communicate with a computer terminal, will receive and treated, RS-485 serial port via real-time transmission to a computer terminal. RS-485[4] interface using a combination of balanced differential drivers and receivers, and anti-common mode capacity enhancement, namely good noise immunity.

\subsection{Computer display terminals}

Computer terminal to switch status information in real-time monitoring and analysis of data, collection and processing of data in real time. Based on real-time and ensure accuracy of the data, the system uses the ADO technology (Mieorsoft Aetivex Data Objeets) technology that is the dynamic data object [5]. ADO is a new interface Micosoft database application development, it has strong features, versatility, high efficiency, occupy less space and so on. In several database access methods in VB, ADO most powerful. ADO interface protocol used in the system database interface software design, the use of ADO to connect [6], ADO data sources between the front and with a minimum number of layers to provide a lightweight, high-performance interface directly with SQL Sevrer database server.

Switch status information computer terminal will switch the receiving means for transmitting real-time analysis, display testing, storage. Among them, the computer display terminal can be a PC or tablet, this can be selected according to the construction site. Display terminal while multi-channel switch status information is analyzed to detect and analyze the test results will be stored in the corresponding database, where "0" indicates the switch to off, "1" for the switch status is closed, "2 "collection device for the switch off state when the switch state acquisition unit is not working properly. Figure 3 is a database to store data. In the display window switch status detection, the staff can observe the corresponding switch working condition, marked with Simplified Chinese are "open" means that the switch to off, "together" indicates switch status to a closed state. "offline "indicates the switch status collection unit is not working properly. Figure 4 is a switch status detection device of a computer terminal display window.

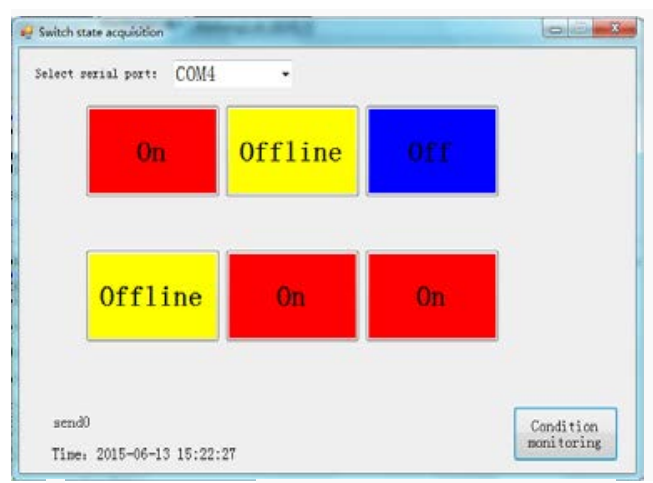

Figure 4. Database to store data

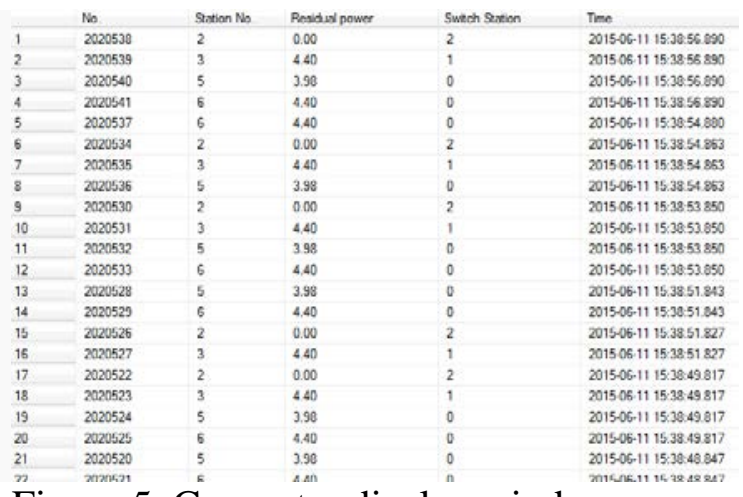

Figure 5. Computer display window

\section{Conclusions}

The program gives the multiplex switch status detection device based on wireless communication can simultaneously detect multiple switch status, which switch status acquisition device and switch status receiving device by way of wireless communication transmission switch status information, switch status receiving device via RS-485 serial cable to communicate with the host computer. To ensure the accuracy of the collection switch status and simplify the circuit structure, it uses software to dither method. The system also has a low power consumption, when the switch status does not change, the acquisition device go into hibernation, and the default is every eight seconds, a self- wake. 
The system has been put into use in the actual plant, after a long test, stable, practical workshop to verify the feasibility of the theory, bringing practical benefits for the workshop production process.

\section{References}

[1] Yin Peng.The design and implementation of remote monitoring system of switch quantity [D]. Xi'an University of Electronic Science and Technology, 2008.

[2] Xin Yan, Chun-Sheng Ma, Chuan-Tao Zheng, Da-Ming Zhang, Multi-channel switch array on the base of triple series-coupled electro-optical polymer microring resonators, Optics \& Laser Technology, Volume 53, December 2013, Pages 9-16.

[3]Imre Szabo, Kalman Mathe, Attila Toth, Andras Czurko, The application of cross-point switch arrays as input selector switch devices for multi-channel electrophysiological experiments, Journal of Neuroscience Methods, Volume 111, Issue 1, 15 October 2001, Pages 75-81.

[4]Chih-Yung Chang, Yu-Chieh Chen, Li-Ling Hung, Sheng-Wen Chang, A novel multi-channel MAC protocol with directional antenna for enhancing spatial reuse and bandwidth utilization in WLANs, Journal of Parallel and Distributed Computing, Volume 69, Issue 10, October 2009, Pages 824-837.

[5]Sandrine Heitz-Spahn, Cross-channel free-riding consumer behavior in a multichannel environment: An investigation of shopping motives, sociodemographics and product categories, Journal of Retailing and Consumer Services, Volume 20, Issue 6, November 2013, Pages 570-578.

[6]HuaJun Huang, Dong Ruan. Single boson realizations of the deformed angular momentum algebra of Wittens types[J]. Chinese Science Bulletin, 2013, Pages 70-76. 